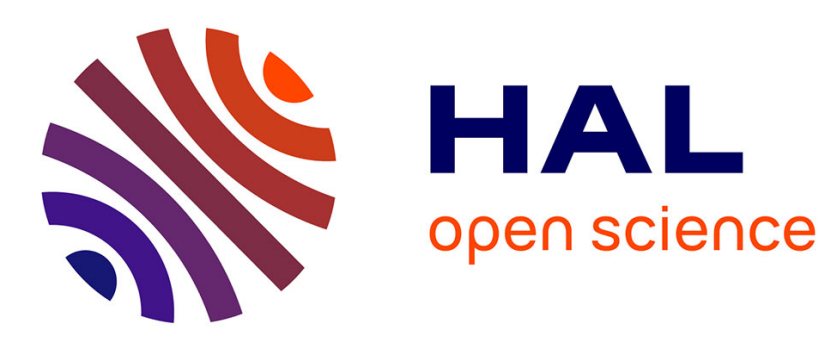

\title{
Consensus, communication and knowledge: an extension with bayesian agents \\ Lucie Ménager
}

\section{To cite this version:}

Lucie Ménager. Consensus, communication and knowledge: an extension with bayesian agents. 2005. halshs-00194381

\section{HAL Id: halshs-00194381 \\ https://shs.hal.science/halshs-00194381}

Submitted on 6 Dec 2007

HAL is a multi-disciplinary open access archive for the deposit and dissemination of scientific research documents, whether they are published or not. The documents may come from teaching and research institutions in France or abroad, or from public or private research centers.
L'archive ouverte pluridisciplinaire HAL, est destinée au dépôt et à la diffusion de documents scientifiques de niveau recherche, publiés ou non, émanant des établissements d'enseignement et de recherche français ou étrangers, des laboratoires publics ou privés. 

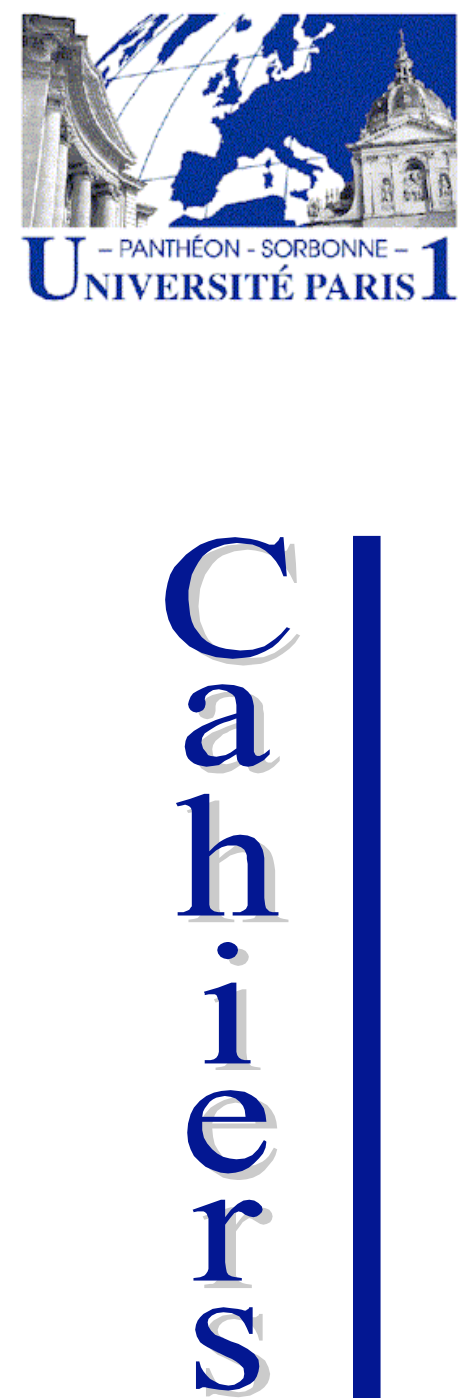

de

la
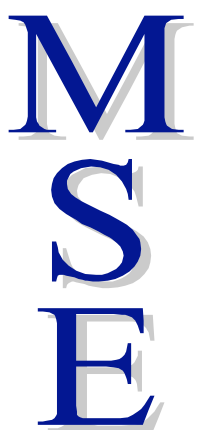

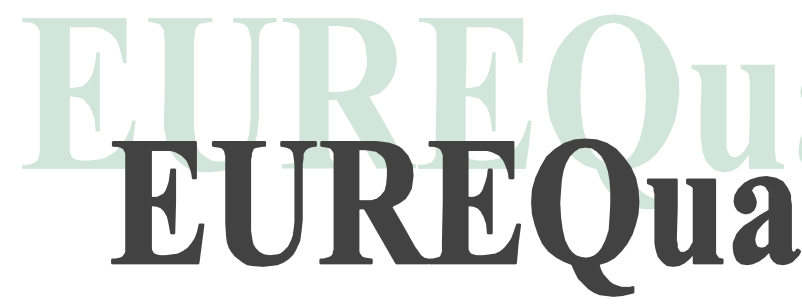

Equipe Universitaire de Recherche en Economie Quantitative - UMR 8594

Consensus, communication and knowledge : an extension with bayesian agents

Lucie MENAGER, EUREQua

2005.31 


\title{
Consensus, Communication and Knowledge: an Extension with Bayesian Agents
}

\author{
Lucie Ménager*†
}

26th January 2006

\begin{abstract}
Parikh and Krasucki [1990] showed that pairwise communication of the value of a function $f$ leads to a consensus about the communicated value if the function $f$ is convex. They showed that union consistency of $f$ may not be sufficient to guarantee consensus in any communication protocol. Krasucki [1996] proved that consensus occurs for any union consistent function if the protocol contains no cycle. We show that if agents communicate their optimal action, namely the action that maximizes their expected utility, then consensus obtains in any fair protocol for any action space.
\end{abstract}

JEL Classification: D82.

Keywords: Consensus, Common knowledge, Pairwise Communication.

\section{Introduction}

Aumann [1976] proved that if two individuals have the same prior beliefs, then common knowledge of their posterior beliefs for an event implies the equality of these posteriors. Geanakoplos and Polemarchakis [1982] extended Aumann's result to a dynamic framework, and showed that communication of posterior beliefs leads to a situation of common knowledge

\footnotetext{
${ }^{*}$ Centre d'Economie de la Sorbonne-CNRS-Université Paris I, 106-112 bld de l'Hôpital, 75013 Paris, E-mail: menager@univ-paris1.fr, Tel: +331440782 12

${ }^{\dagger}$ I thank Jean-Marc Tallon and an anonymous referee for their comments and for help in improving the exposition of the paper. Financial support from the French Ministry of Research (Actions Concertées Incitatives) is gratefully acknowledged.
} 
of these posteriors. Cave [1983] and Bacharach [1985] proved these agreement results considering union consistent ${ }^{1}$ functions more general than posterior beliefs. In all of these settings, communication is public, as achieved e.g. by auctions. Parikh and Krasucki [1990] investigated the case where communication is not public but in pairs. They defined an updating process along which agents communicate with each other, according to a protocol upon which they have agreed beforehand. At each stage one of the agents transmits to another agent the value of a certain function $f$, which depends on the set of states of the world she conceives as possible at that stage. Parikh and Krasucki [1990] showed that two conditions guarantee that eventually, all agents will communicate the same value (a situation we will refer to as a consensus): 1) a fairness condition on the communication protocol, which imposes that every agent has to be sender and receiver of the communication infinitely many times; 2) a convexity condition on the function whose value is communicated. Let $\Omega$ be the set of states of the world. A function $f: 2^{\Omega} \rightarrow \mathbb{R}$ is convex if $\forall X, Y \in 2^{\Omega}$ such that $X \cap Y=\emptyset$, there exists $\alpha \in] 0,1[$ such that $f(X \cup Y)=\alpha f(X)+(1-\alpha) f(Y)$. This condition is satisfied by conditional probabilities for instance, and is more restrictive than Cave's union consistency.

Parikh and Krasucki's convexity condition may not apply in some contexts, as shown in the following example. An individual contemplates buying a car. The set of available decisions is $\{$ buy, not buy $\}$. Suppose that we re-label the decisions in $\mathbb{R}$, with for instance 1 standing for buy and 0 standing for not buy. The convexity condition implies that if $f(X)=0$ and $f(Y)=1$ for some $X, Y$ such that $X \cap Y=\emptyset$, then $f(X \cup Y) \in] 0,1[$, which does not correspond to any decision in $\{b u y, n o t$ buy $\}$. Hence there are some decision spaces for which, even after a re-labelling in $\mathbb{R}$, we may not be able to apply the convexity condition.

Parikh and Krasucki [1990] showed by a counter-example that weak convexity ${ }^{2}$ and union consistency are not sufficient to guarantee that consensus occurs in any fair protocol. Krasucki [1996] investigated what restrictions on the communication protocol should be imposed to guarantee the consensus with any union consistent function. He showed that if the protocol is fair and contains no cycle, then communication of the value of any union consistent function leads to consensus.

\footnotetext{
${ }^{1}$ Let $\Omega$ be the set of states of the world. $f: 2^{\Omega} \rightarrow \mathcal{D}$ is union consistent if $\forall X, Y \in 2^{\Omega}$ such that $X \cap Y=\emptyset$, $f(X)=f(Y) \Rightarrow f(X \cup Y)=f(X)=f(Y)$.

${ }^{2}$ Let $\Omega$ be the set of states of the world. $f: 2^{\Omega} \rightarrow \mathbb{R}$ is weakly convex if $\forall X, Y \in 2^{\Omega}$ such that $X \cap Y=\emptyset$, there exists $\alpha \in[0,1]$ such that $f(X \cup Y)=\alpha f(X)+(1-\alpha) f(Y)$.
} 
In this note, we give a new condition on $f$ for consensus to emerge in any fair communication protocol. This condition is that the function whose values are communicated is the maximizer of a conditional expected utility. Contrary to Parikh and Krasucki's convexity condition, this condition applies to any action space.

Even after an appropriate re-labelling of the image of $f$ in $\mathbb{R}$, the functions we consider may not be representable by weakly convex functions. Furthermore, there exist weakly convex functions that do not obey our condition. Hence the class of functions we look at have a nonempty intersection with the class of weakly convex functions, but there is no inclusion relation among them. On the other hand, for any decision space, the functions we consider are union consistent.

\section{Reaching a consensus}

Let $\Omega$ be a finite set of states of the world. We consider a group of $N$ agents, each of them endowed with a partition $\Pi_{i}$ of $\Omega$. All agents share some prior belief $P$ on $\Omega$. We note $\Pi_{i}(\omega)$ the cell of $\Pi_{i}$ that contains $\omega . \Pi_{i}(\omega)$ is the set of states that $i$ judges possible when state $\omega$ occurs. As in Parikh and Krasucki [1990], agents communicate the value of a function $f: 2^{\Omega} \rightarrow \mathcal{D}$, according to a fair protocol $\operatorname{Pr}$. A protocol is a pair of functions $(s(),. r()):. \mathbb{N} \rightarrow\{1, \ldots, N\}^{2}$ where $s(t)$ stands for the sender and $r(t)$ the receiver of the communication which takes place at time $t$. A protocol is fair $^{3}$ if no participant is blocked from the communication, that is if every agent is a sender and a receiver infinitely many times, and everyone receives information from every other, possibly indirectly, infinitely many times. Except fairness, we do not make any assumption on the protocol. We assume that $\mathcal{D}$ can be any compact subset of a topological space.

Agents share a common payoff function $U: \mathcal{D} \times \Omega \rightarrow \mathbb{R}$, which depends on the chosen action $d \in \mathcal{D}$ and on the realized state of the world. We assume that $U(., \omega)$ is continuous on $\mathcal{D}$ for all $\omega$. What is communicated by an agent is the action that maximizes her expected utility, computed with respect to the common belief $P$. In order to avoid indifference cases, we make the assumption that given any event, all actions have different expected utility conditional on

\footnotetext{
${ }^{3}$ Given a protocol $(s(t), r(t))$ consider the directed graph whose vertices are the participants $\{1, \ldots, N\}$ and such that there is an edge from $i$ to $j$ iff there are infinitely many $t$ such that $s(t)=i$ and $r(t)=j$. The protocol is fair if the graph above is strongly connected.
} 
this event. That is to say given an event $F \subseteq \Omega, \forall d, d^{\prime} \in \mathcal{D}, E(U(d,) \mid F.) \neq E\left(U\left(d^{\prime},.\right) \mid F\right)$. Without this assumption, the set of maximizing actions of an agent may not be a singleton, and we would have to specify the way agents choose between indifferent actions.

The function $f: 2^{\Omega} \rightarrow \mathcal{D}$ is then defined by:

$$
\forall E \subseteq \Omega, f(E)=\operatorname{argmax}_{d \in \mathcal{D}} \mathrm{E}(U(d, .) \mid E)
$$

Suppose now that $\operatorname{Pr}$ is some given protocol. The set of possible states for an agent $i$ at time $t$ if the state of the world is $\omega$ is denoted $C_{i}(\omega, t)$ and is defined by the following recursive process:

$$
\begin{aligned}
& C_{i}(\omega, 0)=\Pi_{i}(\omega) \\
& C_{i}(\omega, t+1)=C_{i}(\omega, t) \cap\left\{\omega^{\prime} \in \Omega \mid f\left(C_{s(t)}\left(\omega^{\prime}, t\right)\right)=f\left(C_{s(t)}(\omega, t)\right)\right\} \text { if } i=r(t), \\
& C_{i}(\omega, t+1)=C_{i}(\omega, t) \text { otherwise. }
\end{aligned}
$$

The next result states that for all $\omega, f\left(C_{i}(\omega, t)\right)$ has a limiting value which does not depend on $i$.

Theorem 1 There is a $T \in \mathbb{N}$ such that for all $\omega$, $i$, and all $t, t^{\prime} \geq T, C_{i}(\omega, t)=C_{i}\left(\omega, t^{\prime}\right)$. Moreover, if the protocol is fair, then for all $i, j$, for all $\omega, f\left(C_{i}(\omega, T)\right)=f\left(C_{j}(\omega, T)\right)$.

We now discuss the properties of the function $f$ defined as the argmax of an expected utility. First, $f$ is clearly union consistent for any action space. Second, $f$ may not be representable by a weakly convex function, namely a one to one function $g: \mathcal{D} \rightarrow \mathbb{R}$ may fail to exist such that $g \circ f$ is weakly convex. If such a function $g$ were to exist, learning and consensus properties of $f$ and $g \circ f$ would be the same. Therefore, the functions $f$ we consider would be particular weakly convex functions, for which consensus obtains in any fair protocol. We show that it is not the case with the following counter example.

Consider the case where $\Omega=\{1,2,3,4\}, \mathcal{D}=\{a, b, c\}, P$ is uniform $(P(\omega)=1 / 4 \forall \omega)$ and the utility function $U$ is defined by:

$$
\begin{aligned}
& U(a, 1)=1, U(a, 2)=0, U(a, 3)=1, U(a, 4)=0 \\
& U(b, 1)=0, U(b, 2)=1, U(b, 3)=2 / 3, U(b, 4)=2 / 3 \\
& U(c, 1)=2 / 3, U(c, 2)=2 / 3, U(c, 3)=0, U(c, 4)=1
\end{aligned}
$$


We have in particular:

$$
f(\{1\})=a, f(\{2\})=b, f(\{3\})=a, f(\{4\})=c, f(\{1,2\})=c, f(\{3,4\})=b
$$

For any one to one function $g: \mathcal{D} \rightarrow \mathbb{R}$, six cases are possible. We show that in each case, $g \circ f$ is not weakly convex.

1. If $g(a)<g(b)<g(c)$, then $g \circ f(\{1\})<g \circ f(\{2\})<g \circ f(\{1,2\})$.

2. If $g(a)<g(c)<g(b)$, then $g \circ f(\{3\})<g \circ f(\{4\})<g \circ f(\{3,4\})$.

3. If $g(b)<g(a)<g(c)$, then $g \circ f(\{3,4\})<g \circ f(\{3\})<g \circ f(\{4\})$.

4. If $g(b)<g(c)<g(a)$, then $g \circ f(\{3,4\})<g \circ f(\{4\})<g \circ f(\{3\})$.

5. If $g(c)<g(a)<g(b)$, then $g \circ f(\{1,2\})<g \circ f(\{1\})<g \circ f(\{2\})$.

6. If $g(c)<g(b)<g(a)$, then $g \circ f(\{1,2\})<g \circ f(\{2\})<g \circ f(\{1\})$.

Finally, there exist weakly convex functions that cannot be defined as the argmax of an expected utility. Such an example can be found in Parikh and Krasucki [1990, p 185]: they exhibit a weakly convex function $f$ such that consensus may fail to occur in some protocols. It can be shown easily that it is not possible to find a utility function $U$ and a probability $P$ such that this function $f$ is the argmax of the conditional expectation of $U$.

\section{References}

[1] Aumann R. J., [1976], Agreeing to Disagree, The Annals Of Statistics, 4, 1236-1239.

[2] Bacharach M., [1985], Some Extensions of a Claim of Aumann in an Axiomatic Model of Knowledge, Journal of Economic Theory, 37, 167-190.

[3] Cave J., [1983], Learning To Agree, Economics Letters, 12, 147-152.

[4] Geanakoplos J., Polemarchakis H., [1982], We Can't Disagree Forever, Journal of Economic Theory, 28, 192-200.

[5] Krasucki P., [1996], Protocols Forcing Consensus, Journal of Economic Theory, 70, 266272. 
[6] Parikh R., Krasucki P., [1990], Communication, Consensus and Knowledge, Journal of Economic Theory, 52, 178-189.

\section{Proof: [Theorem 1]}

1) As $\Omega$ is finite, the first part of the theorem is evident. In the sequel, we will note $C_{i}(\omega)$ the limiting value of $C_{i}(\omega, t)$, and $C_{i}$ the information partition of agent $t$ at equilibrium.

2) As in Parikh and Krasucki [1990], we prove the second part of the theorem for $N=3$ and for a "round-robin protocol", namely such that for all $t, s(t)=t \bmod 3$ and $r(t)=(t+1)$ mod 3. Note that this is sufficient to prove the theorem for any fair protocol. Our argument only uses the fact that we are able to find a chain $t_{1}<t_{2}<\cdots<t_{p}$, with $T \leq t_{1}$, such that: $(a) s\left(t_{1}\right)=1,(b)$ the receiver at $t_{j}$ is the sender at $t_{j+1},(c)$ the chain passes through all participants, finally returning to 1 . This is implied by the fact that the protocol is fair.

Let $M_{i j}$ be the partition of common knowledge among agents $i$ and $j$ at equilibrium, that is $M_{i j}$ is the finest partition of $\Omega$ such that $\forall \omega, C_{i}(\omega) \subseteq M_{i j}(\omega)$ and $C_{j}(\omega) \subseteq M_{i j}(\omega)$. By consequence, $\forall \omega, M_{i j}(\omega)$ is a disjoint union of cells of $C_{i}$ and a disjoint union of cells of $C_{j}$. $\sum_{C_{i}(k) \subseteq M_{i j}(\omega)}$ will denote the sum on all cells of $C_{i}$ composing $M_{i j}(\omega)$.

At equilibrium, agent 1 communicates her optimal action to agent 2 , agent 2 communicates her optimal action to agent 3 and agent 3 communicates her optimal action to agent 1 . By consequence, the action taken by agent 1 is common knowledge among 1 and 2 . Hence we have for all $\omega$ :

$$
M_{12}(\omega) \subseteq\left\{\omega^{\prime} \in \Omega \mid f\left(C_{1}\left(\omega^{\prime}\right)\right)=f\left(C_{1}(\omega)\right)\right\}
$$

As $M_{12}(\omega)$ is a disjoint union of cells of $C_{1}$, union consistency of $f$ implies that $f\left(M_{12}(\omega)\right)=f\left(C_{1}(k)\right)$ $\forall k \in M_{12}(\omega)$.

- Result $1 E\left(U\left(f\left(M_{12}(\omega)\right),.\right) \mid M_{12}(\omega)\right)=E\left[E\left(U\left(f\left(C_{1}(\cdot)\right),.\right) \mid C_{1}(\cdot)\right) \mid M_{12}(\omega)\right]$

Proof: For all $\omega^{\prime} \in M_{12}(\omega), f\left(C_{1}\left(\omega^{\prime}\right)\right)=f\left(M_{12}(\omega)\right)$. Then $E\left[E\left(U\left(f\left(C_{1}(\cdot)\right),.\right) \mid C_{1}().\right) \mid M_{12}(\omega)\right]=$ $E\left[E\left(U\left(f\left(M_{12}(\omega)\right),.\right) \mid C_{1}(\cdot)\right) \mid M_{12}(\omega)\right]$. As $M_{12}$ is coarser than $C_{1}$, the law of iterated expectations implies that $E\left[E\left(U\left(f\left(M_{12}(\omega)\right),.\right) \mid C_{1}().\right) \mid M_{12}(\omega)\right]=E\left(U\left(f\left(M_{12}(\omega)\right),.\right) \mid M_{12}(\omega)\right]$.

- Result $2 E\left(U\left(f\left(M_{12}(\omega)\right),.\right) \mid M_{12}(\omega)\right) \leq \sum_{C_{2}(k) \subseteq M_{12}(\omega)} \frac{P\left(C_{2}(k)\right)}{P\left(M_{12}(\omega)\right)} E\left(U\left(f\left(C_{2}(k)\right),.\right) \mid C_{2}(k)\right)$ 
Proof: By definition, $\forall k \in M_{12}(\omega)$ we have:

$$
E\left(U\left(f\left(M_{12}(\omega)\right), .\right) \mid C_{2}(k)\right) \leq E\left(U\left(f\left(C_{2}(k)\right), .\right) \mid C_{2}(k)\right)
$$

It implies that:

$$
\sum_{C_{2}(k) \subseteq M_{12}(\omega)} P\left(C_{2}(k)\right) E\left(U\left(f\left(M_{12}(\omega)\right), .\right) \mid C_{2}(k)\right) \leq \sum_{C_{2}(k) \subseteq M_{12}(\omega)} P\left(C_{2}(k)\right) E\left(U\left(f\left(C_{2}(k)\right), .\right) \mid C_{2}(k)\right)
$$

that is:

$$
P\left(M_{12}(\omega)\right) E\left(U\left(f\left(M_{12}(\omega)\right), .\right) \mid M_{12}(\omega)\right) \leq \sum_{C_{2}(k) \subseteq M_{12}(\omega)} P\left(C_{2}(k)\right) E\left(U\left(f\left(C_{2}(k)\right), .\right) \mid C_{2}(k)\right) \square
$$

- Result $3 \forall i, j, E\left[E\left(U\left(f\left(C_{i}(\cdot)\right),.\right) \mid C_{i}(\cdot)\right)\right]=E\left[E\left(U\left(f\left(C_{j}(\cdot)\right),.\right) \mid C_{j}(\cdot)\right)\right]$

Proof:

$$
E\left[E\left(U\left(f\left(C_{1}(\cdot)\right), .\right) \mid C_{1}(\cdot)\right)\right]=\sum_{M_{12}(\omega) \subseteq \Omega} P\left(M_{12}(\omega) E\left[E\left(U\left(f\left(C_{1}(\cdot)\right), .\right) \mid C_{1}(\cdot)\right) \mid M_{12}(\omega)\right]\right.
$$

Yet by results $\mathbf{1}$ and $\mathbf{2}$, we have

$$
P\left(M_{12}(\omega)\right) E\left[E\left(U\left(f\left(C_{1}(\cdot)\right), .\right) \mid C_{1}(\cdot)\right) \mid M_{12}(\omega)\right] \leq \sum_{C_{2}(k) \subseteq M_{12}(\omega)} P\left(C_{2}(k)\right) E\left(U\left(f\left(C_{2}(k)\right), .\right) \mid C_{2}(k)\right)
$$

Then

$$
\begin{aligned}
E\left[E\left(U\left(f\left(C_{1}(.)\right), .\right) \mid C_{1}(.)\right)\right] & \leq \sum_{M_{12}(\omega) \subseteq \Omega} \sum_{C_{2}(k) \subseteq M_{12}(\omega)} P\left(C_{2}(k)\right) E\left(U\left(f\left(C_{2}(k)\right), .\right) \mid C_{2}(k)\right) \\
& =\sum_{C_{2}(k) \subseteq \Omega} P\left(C_{2}(k)\right) E\left(U\left(f\left(C_{2}(k)\right), .\right) \mid C_{2}(k)\right) \\
& =E\left[E\left(U\left(f\left(C_{2}(\cdot)\right), .\right) \mid C_{2}(\cdot)\right)\right]
\end{aligned}
$$

Applying the same reasoning, we get

$$
E\left[E\left(U\left(f\left(C_{2}(\cdot)\right), .\right) \mid C_{2}(\cdot)\right)\right] \leq E\left[E\left(U\left(f\left(C_{3}(\cdot)\right), .\right) \mid C_{3}(\cdot)\right)\right]
$$

and

$$
E\left[E\left(U\left(f\left(C_{3}(\cdot)\right), .\right) \mid C_{3}(\cdot)\right)\right] \leq E\left[E\left(U\left(f\left(C_{1}(\cdot)\right), .\right) \mid C_{1}(\cdot)\right)\right]
$$

Hence $E\left[E\left(U\left(f\left(C_{i}(\cdot)\right),.\right) \mid C_{i}(\cdot)\right)\right]=E\left[E\left(U\left(f\left(C_{j}(\cdot)\right),.\right) \mid C_{j}(\cdot)\right)\right]$ for all $i, j$. 
- Result 4 For all $\omega \in \Omega$, we have

$E\left(U\left(f\left(C_{1}(\omega)\right),.\right) \mid C_{2}(\omega)\right)=E\left(U\left(f\left(C_{2}(\omega)\right),.\right) \mid C_{2}(\omega)\right)$

$E\left(U\left(f\left(C_{2}(\omega)\right),.\right) \mid C_{3}(\omega)\right)=E\left(U\left(f\left(C_{3}(\omega)\right),.\right) \mid C_{3}(\omega)\right)$

$E\left(U\left(f\left(C_{3}(\omega)\right),.\right) \mid C_{1}(\omega)\right)=E\left(U\left(f\left(C_{1}(\omega)\right),.\right) \mid C_{1}(\omega)\right)$

Proof:

By Result 3, the inequality can not be strict in Result 2. Then we have:

$$
P\left(M_{12}(\omega)\right) E\left(U\left(f\left(M_{12}(\omega)\right), .\right) \mid M_{12}(\omega)\right)=\sum_{C_{2}(k) \subseteq M_{12}(\omega)} P\left(C_{2}(k)\right) E\left(U\left(f\left(C_{2}(k)\right), .\right) \mid C_{2}(k)\right)
$$

By definition, $E\left(U\left(f\left(C_{1}(k)\right),.\right) \mid C_{2}(k)\right) \leq E\left(U\left(f\left(C_{2}(k)\right),.\right) \mid C_{2}(k)\right)$ for all $k \in M_{12}(\omega)$.

If $\exists k$ such that $E\left(U\left(f\left(C_{1}(k)\right),.\right) \mid C_{2}(k)\right)<E\left(U\left(f\left(C_{2}(k)\right),.\right) \mid C_{2}(k)\right)$, then

$$
\sum_{C_{2}(k) \subseteq M_{12}(\omega)} P\left(C_{2}(k)\right) E\left(U\left(f\left(C_{1}(k)\right), .\right) \mid C_{2}(k)\right)<\sum_{C_{2}(k) \subseteq M_{12}(\omega)} P\left(C_{2}(k) E\left(U\left(f\left(C_{2}(k)\right), .\right) \mid C_{2}(k)\right)\right.
$$

that is:

$$
P\left(M_{12}(\omega)\right) E\left(U\left(f\left(M_{12}(\omega)\right), .\right) \mid M_{12}(\omega)\right)<\sum_{C_{2}(k) \subseteq M_{12}(\omega)} P\left(C_{2}(k) E\left(U\left(f\left(C_{2}(k)\right), .\right) \mid C_{2}(k)\right)\right.
$$

which is a contradiction.

Hence we have $E\left(U\left(f\left(C_{1}(k)\right),.\right) \mid C_{2}(k)\right)=E\left(U\left(f\left(C_{2}(k)\right),.\right) \mid C_{2}(k)\right)$ for all $k \in M_{12}(\omega)$. As it is true for all $\omega$, we have $E\left(U\left(f\left(C_{1}(k)\right),.\right) \mid C_{2}(k)\right)=E\left(U\left(f\left(C_{2}(k)\right),.\right) \mid C_{2}(k)\right)$ for all $k \in \Omega$. The same reasoning applies for 2,3 and 3,1 .

From Result 4 and the assumption that all actions bring different expected utilities, we have

$$
f\left(C_{1}(\omega)\right)=f\left(C_{2}(\omega)\right)=f\left(C_{3}(\omega)\right) \forall \omega \in \Omega
$$

